\title{
(อ) \\ Diagnosing ALS: the Gold Coast criteria and the role of EMG
}

\author{
Martin R Turner ํ) , UK MND Clinical Studies Group
}

Correspondence to

Professor Martin R Turner, Nuffield Department of Clinical Neurosciences, University of Oxford Medical Sciences Division, Oxford OX3 9DU, UK: martin.turner@ndcn.ox.ac.uk

Accepted 26 October 2021

Check for updates

(C) Author(s) (or their employer(s)) 2022. Re-use permitted under CC BY Published by BMJ.

To cite: Turner MR, Pract Neurol Epub ahead of print: [please include Day Month Year]. doi:10.1136/ practneurol-2021-003256
In September 2019, a group of international neurologists gathered in Gold Coast, Australia, to deconstruct the diagnostic process for amyotrophic lateral sclerosis (ALS) and to try to simplify it. A proposal for revised diagnostic criteria emerged ${ }^{1}$ (box 1) and the initial experience of their application has been positive. ${ }^{2-5}$ The diagnosis remains fundamentally clinical, and it is timely to reflect on the adjunctive role of electromyography (EMG).

Although motor neurone disease (MND) is undeniably clinically heterogeneous, there is a unifying molecular pathology in $97 \%$ of cases, namely cytoplasmic phosphorylated aggregates of the $43 \mathrm{kDa}$ transactive response DNAbinding protein, TDP-43. Since Charcot's pivotal clinical observations of the simultaneous occurrence of upper motor neurone (UMN) and lower motor neurone (LMN) involvement defining the the most common phenotype of ALS, a broader spectrum of involvement is now recognised clinically and pathologically. ${ }^{6}$ At both LMN and UMN clinical extremes, the rate of disease progression is notably slower, but there is no simplistic relationship between the site of onset, the combination of UMN versus $\mathrm{LMN}$ signs or rate of disease progression. The historical term progressive muscular atrophy was used to describe the extreme of clinical LMN involvement, but histopathological ${ }^{7}$ and neuroimaging ${ }^{8}$ studies have shown that such cases have subclinical corticospinal tract and wider brain involvement, and the modern use of the term 'LMN-predominant ALS' reflects this. The other extreme is also a spectrum of 'UMN-predominant ALS' in which LMN signs are not clinically obvious until a few years after symptom onset. A minority of these cases, and less than 3\% of all MND, can be confidently demarcated as primary lateral sclerosis by virtue of a dramatically slower rate of progression associated with marked spasticity as well as lack of LMN involvement. ${ }^{9}$ The clinicopathological overlap of ALS with frontotemporal dementia has further extended Charcot's definition, so that ALS may now be considered a system degeneration variably penetrating the motor cortex and its spinal and cerebral connectome.

The original diagnostic criteria defined at El Escorial, Spain, in 1990 and their subsequent 1998 revision at Airlie House, USA, and in 2006 at Awaji-shima, Japan, employed categories but were created very much with therapeutic trials in mind. Categories were based on the number of body regions (bulbar, cervical, thoracic and lumbosacral) with simultaneous UMN as well as LMN signs. The terms used in the various iterations, included some or all of: 'suspected', 'possible', 'probable', 'probable laboratory-supported' and 'definite' ALS. These categories have been shown to have little independent prognostic value $^{10}$ and patients with MND deemed to have 'insufficient' UMN signs clinically were denied entry to trials, despite similar rates of disability progression as 'probable' or 'definite' cases. Individuals frequently die from MND never having evolved from their 'suspected', 'possible' or 'probable' categories. It is biologically interesting how little clinical evolution there is between these categories, despite a wide range of disability progression rates common to all of them. Speculatively, the proportions of UMN versus LMN involvement might reflect an individual's premorbid nervous system architecture to some extent. Most problematic of all, however, was the often psychologically distressing consequences for patients in being told they had anything other than 'definite' ALS, implying persistent diagnostic doubt where there was usually none.

EMG has been unrivalled to date in providing evidence of subclinical LMN 


\section{Box 1}

The Gold Coast criteria for the diagnosis of amyotrophic lateral sclerosis

1. Progressive motor impairment documented by history or repeated clinical assessment, preceded by normal motor function,

AND

2. The presence of upper ${ }^{*}$ and lowert motor neurone dysfunction in at least ONE body region $¥$, with:

- upper and lower motor neurone dysfunction noted in the same body region if only one region is involved,

- or lower motor neurone dysfunction in at least TWO body regions,

AND

3. Investigations§ excluding other disease processes.

a. Increased deep tendon reflexes, including the presence of a reflex in a clinically weak and wasted muscle, or spread to adjacent muscles.

b. Presence of pathological reflexes, including Hoffman sign, Babinski sign, crossed adductor reflex, or snout reflex.

c. Increase in velocity-dependent tone (spasticity).

d. Slowed, poorly coordinated voluntary movement, not attributable to weakness of lower motor neurone origin or Parkinsonian features.

a. Evidence of chronic neurogenic change, defined by large motor unit potentials of increased duration and/or increased amplitude (with polyphasia), and motor unit instability regarded as supportive but not obligatory evidence, and

b. Evidence of ongoing denervation, including fibrillation potentials or positive sharp waves, or fasciculation potentials.

* Upper motor neurone dysfunction implies at least one of the following:

tLower motor neurone dysfunction in a given muscle requires either:

Clinical examination evidence of muscle weakness and muscle wasting, or

EMG abnormalities that must include both:

$¥$ \#ody regions are defined as bulbar, cervical, thoracic and lumbosacral. To be classified as an involved region with respect to lower motor neurone involvement, there must be abnormalities in TWO limb muscles innervated by different roots and nerves, or ONE bulbar muscle, or one thoracic muscle, either by clinical examination or by electromyography (EMG).

$\S$ The appropriate investigations depend on the clinical presentation, and may include nerve conduction studies and needle EMG, MR or other imaging, biofluid studies, or other modalities as clinically necessary.

involvement, although with persistent debate as to what changes are pathognomic for neurodegenerative denervation. Importantly however, EMG is far from $100 \%$ sensitive and highly operator dependent. The sensitivity shortfall is most often apparent in cases of UMN-predominant ALS, particularly those with bulbar onset. Historically (but no longer usefully) termed progressive bulbar palsy, such cases are commonly found in women aged over 70 years. Symptoms may remain relatively confined to the bulbar territory for more than a year before wider generalisation. There is often a lack of tongue as well as limb EMG findings. In such cases, over-reliance on EMG as a diagnostic test for MND leads to avoidable diagnostic delay. This may impact timely care planning, for example, gastrostomy, access to licensed disease-modifying drugs like riluzole and trials of new candidate drugs.

The Gold Coast group recognised rapid developments in new potential biomarkers of subclinical UMN degeneration, for example, blood neurofilament concentrations, diffusion tensor imaging, transcranial magnetic stimulation; and LMN degeneration, for example, muscle ultrasound detection of subclinical fasciculation. Such biomarkers might lead to further refinement of these criteria. In the longer-term, a TDP-43 assay might even form the basis for wider population screening. For now, the immediate hope is that the Gold Coast criteria will remove the distress and confusion caused by the historical diagnostic category terms. The new criteria still have therapeutic trials very much at the forefront of thinking. Replacing site of symptom onset, only one factor influencing overall prognosis, with a multiaxial classification based on rate of progression and the pattern of progression of disability might ultimately provide a more meaningful basis for stratification.

For the individual with progressive weakness, the diagnosis is 'ALS', or 'not ALS'. EMG remains valuable in providing evidence of clinically occult LMN involvement but is an adjunct to a history and examination-led diagnostic process. Finally, a cornerstone of good medical practice is that any diagnosis, but especially a life-shortening one, should be followed by an outline of proposed care. We suggest that the diagnosis of ALS should be conveyed by a clinician with knowledge of that plan.

\section{Further reading}

- Vucic S, Ferguson TA, Cummings C, Hotchkin MT, Genge A, Glanzman R, et al. Gold Coast diagnostic criteria: implications for ALS diagnosis and clinical trial enrollment. Muscle \& Nerve. 2021. 
Acknowledgements We would like to thank the UK MND CSG coordinator Ms Stacy Young and Motor Neurone Disease Association for their active engagement with the group's activities.

Collaborators Named members of the UK MND Clinical Studies Group reviewed and edited the manuscript: Ammar Al-Chalabi (King's College London); Idris Baker (Swansea Bay University Health Board); Andrew Barritt (University Hospitals Sussex NHS Foundation Trust); Siddharthan Chandran (University of Edinburgh); Suresh Kumar Chhetri (Lancashire Teaching Hospitals NHS Foundation Trust); Richard Davenport (Department of Clinical Neurosciences, Royal Hospital for Children and Young People and Clinical Neurosciences, Royal Infirmary of Edinburgh); John Ealing (Manchester Centre for Clinical Neurosciences, Salford); George H. Gorrie (Queen Elizabeth University Hospital, Glasgow); Hisham Hamdalla (Manchester Centre for Clinical Neurosciences, Salford); C Oliver Hanemann (Peninsula Medical School, University of Plymouth); Tom Lambert (University Hospitals North Midlands); Karen E. Morrison (Faculty of Medicine, Health and Life Sciences, Queen's University Belfast); Richard W. Orrell (Institute of Neurology, London); Suvankar Pal (University of Edinburgh); Aleksander Radunovic (Royal London Hospital); Muhammad Rafiq (Norfolk \& Norwich University Hospitals NHS Foundation Trust); Kevin Talbot (University of Oxford, Nuffield Department of Clinical Neurosciences); Tim Williams (Department of Neurology, Royal Victoria Infirmary, Newcastle); Carolyn A. Young (Walton Centre NHS Foundation Trust).

Contributors MRT conceived and drafted the manuscript on behalf of the UK MND Clinical Studies Group.

Funding The authors have not declared a specific grant for this research from any funding agency in the public, commercial or not-for-profit sectors.

Competing interests None declared.

Patient consent for publication Not applicable.

Provenance and peer review Not commissioned; externally reviewed by Andrew Chancellor, Tauranga, New Zealand.

Open access This is an open access article distributed in accordance with the Creative Commons Attribution 4.0
Unported (CC BY 4.0) license, which permits others to copy, redistribute, remix, transform and build upon this work for any purpose, provided the original work is properly cited, a link to the licence is given, and indication of whether changes were made. See: https://creativecommons.org/licenses/by/4.0/.

ORCID iD

Martin R Turner http://orcid.org/0000-0003-0267-3180

\section{REFERENCES}

1 Shefner JM, Al-Chalabi A, Baker MR, et al. A proposal for new diagnostic criteria for ALS. Clin Neurophysiol 2020;131:1975-8.

2 Vucic S, Ferguson TA, Cummings C, et al. Gold Coast diagnostic criteria: implications for ALS diagnosis and clinical trial enrollment. Muscle Nerve 2021;64:532-7.

3 Shen D, Yang X, Wang Y, et al. The Gold Coast criteria increases the diagnostic sensitivity for amyotrophic lateral sclerosis in a Chinese population. Transl Neurodegener 2021;10:28.

4 Pugdahl K, Camdessanché J-P, Cengiz B, et al. Gold Coast diagnostic criteria increase sensitivity in amyotrophic lateral sclerosis. Clin Neurophysiol 2021. doi:10.1016/j. clinph.2021.08.014. [Epub ahead of print: 08 Sep 2021].

5 Hannaford A, Pavey N, van den Bos M, et al. Diagnostic utility of gold Coast criteria in amyotrophic lateral sclerosis. Ann Neurol 2021;89:979-86.

6 Turner MR. The reunification of amyotrophic lateral sclerosis. J Neurol Neurosurg Psychiatry 2019;90:122-3.

7 Ince PG, Evans J, Knopp M, et al. Corticospinal tract degeneration in the progressive muscular atrophy variant of ALS. Neurology 2003;60:1252-8.

8 Rosenbohm A, Müller H-P, Hübers A, et al. Corticoefferent pathways in pure lower motor neuron disease: a diffusion tensor imaging study. J Neurol 2016;263:2430-7.

9 Turner MR, Talbot K. Primary lateral sclerosis: diagnosis and management. Pract Neurol 2020;20:262-9.

10 Traynor BJ, Codd MB, Corr B, et al. Clinical features of amyotrophic lateral sclerosis according to the El Escorial and Airlie house diagnostic criteria. Arch Neurol 2000;57:1171-6. 